\title{
Direct Digital Manufacturing of Nanocomposites
}

\author{
Saeed D. Mohan ${ }^{1,2, a}$, Meruyert Nazhipkyzy ${ }^{2,3, b}$, Pedro Carreira ${ }^{1, c}$, \\ Cyril dos Santos ${ }^{1, \mathrm{~d}}$, Fred J. Davis ${ }^{2, \mathrm{e}}$, Artur Mateus ${ }^{1, \mathrm{f}}$ and Geoffrey R Mitchell ${ }^{1, \mathrm{~g}^{*}}$
}

${ }^{1}$ Centre for Rapid and Sustainable Product Development, Polytechnic Institute of Leiria, Portugal

${ }^{2}$ Department of Chemistry, University of Reading, Whiteknights, RG6 6AD, UK

${ }^{3}$ Institute of Combustion Problems, Al-Farabi Kazah National University Almaty, Kazakhstan

as.d.mohan@reading.ac.uk, bmeruert82@mail.ru, cpedro.s.carreira@ipleiria.pt,

dcyril.santos@ipleiria.pt, ef.j.davis@reading.ac.uk, fartur.mateus@ipleiria.pt,

ggeoffrey.mitchell@ipleiria.pt

${ }^{*}$ corresponding author

Keywords: Additive manufacturing, carbon nanotubes, electrospinning, stereo-lithography

\begin{abstract}
Additive manufacturing has surged in popularity as a route to designing and preparing functional parts. Depending on the parts function, certain attributes such as high mechanical performances may be desired. We develop a route for improving the mechanical properties of polymer devices, fabricated through additive manufacturing by combining electrospinning and stereo-lithography into one automated process. This process utilises the impressive mechanical properties of carbon nanotubes by encapsulating and aligning them in electrospun fibres. Composite fibres will be incorporated into polymer resins prepared with stereo-lithography, thereby providing resins that benefit from the composite fibres properties, enhancing their overall mechanical properties.
\end{abstract}

\section{Introduction}

There has been a huge surge of research in the area of additive manufacturing (AM) over the last few years. The technique is very versatile and has a wide array of applications ranging from biomedical to industrial areas. The methods used in AM range from thermal extrusion where a polymer is melted, extruded and deposited in a layer by layer process; granular processing through melting powder layers using for example, a laser, and photo-polymerisation where selective areas of a liquid, photo-curable polymer is exposed to electromagnetic radiation to induce polymerisation and solidification [1]. Through the use of computer aided design (CAD), the additive manufacturing technique allows for the manufacturing of complex shaped parts. Depending on the function of the part, certain properties may be required for the material being engineered, for example enhanced electrical, flame retardant or mechanical properties might be desirable. One method for improving properties of materials is through the addition of a second material, such as a nanoparticle filler. Various examples are present in the literature where nanoparticles with a high aspect ratio such as carbon nanotubes [2], glass fibre [3] and nanocellulose [4]all boost the mechanical properties of a polymeric material. Nanoparticulates such as those listed above, exhibit enhanced properties parallel to their long axis and therefore in order to gain the full benefit of these properties alignment is desirable. However, alignment of nanoparticles presents a particular challenge due to their small scale nature and their inherent tendency to agglomerate due to strong intermolecular (Van der Waal) forces. Various techniques have been researched in order to achieve alignment with methods varying from growth of nanoparticulates in an aligned manner, application of a shear force or the use of electric and magnetic fields [5]. The methodology that we use in this article involves encapsulation within a fibrillar structure, where the process of drawing out and enclosing into a narrow diameter fibre can result in the nanoparticles alignment. One of the simplest and most 
widely researched areas of fibre production over the past decade has been electrospinning, the technique we intend to take full advantage of here.

The electrospinning technique readily produces microscale to nanoscale sized polymer fibres, through the application of a high voltage to a polymer solution or melt contained within a spinneret. The high voltage induces a deformation of the droplet at the needle tip from which a polymer jet is extruded and stretched towards a grounded target electrode. On transit from the spinneret tip to the collector, the jet is stretched down to a micro- to nano- scale polymer fibre and solvent is removed or solidification occurs depending on if the process began with a solution or molten state, thereby leaving a solid polymer fibre on the collector [6].

Electrospun fibres themselves have unique properties owing to their size and high aspect ratio, much similar to the nanoparticulates discussed earlier. During the spinning process the jet undergoes a shearing force and a narrowing in which the nanoparticulates can be aligned and encapsulated within the fibre as it forms. These fibres have the potential to be used in composite materials to aid in various aspects such as mechanical reinforcement. For example, Shinde et al. were able to incorporate electrospun fibres into a resin to improve inter laminar shear stresses of glass fibre composites [7].

In this paper we describe a route to prepare aligned arrays of multi walled carbon nanotubes and incorporate them in the additive manufacturing process through a combination of electrospinning and stereo-lithography.

\section{Experimental}

Electrospinning. Aqueous (deionised water) solutions/suspensions were prepared by weight with loadings of MWCNTs corresponding to $0,0.5 \%, 1 \%, 3 \%$ and $5 \%$ wt of nanotubes in the final fibre matt. $1 \%$ wt Triton X-100 $(\sim 16 \%$ wt in final fibres) was added to the solutions to aid the dispersion of the nanotubes as described in reference [8]. Electrospinning was conducted at a supply voltage of $15 \mathrm{kV}$ and a needle tip to collector distance of $15 \mathrm{~cm}$, with fibres collected onto an aluminium foil substrate placed over a rotating collector.

Scanning electron microscopy (SEM) was performed on sections of the fibre matts produced. The microscopy was performed using a Cambridge Instruments SEM360 in high vacuum mode with an accelerating voltage of $20 \mathrm{kV}$. Fibres were coated with gold prior to examination. Fibres were selected at random from the micrographs obtained and measurements made using the software ImageJ. Transmission electron microscopy (TEM) of the fibres was performed using a Philips CM20 at an operating voltages of $200 \mathrm{kV}$ with samples prepared on a copper TEM grid, 300 mesh. Samples were prepared for TEM by electrospinning directly onto the grid which was secured onto the foil substrate.

Fig. 1a establishes that the production of well aligned, parallel arrays of electrospun fibres containing nanoparticles is possible with a rotating collection speed giving a tangential velocity of 7 $\mathrm{m} / \mathrm{s}$ [8]. Measurements of fibres displayed in Fig. 1a reveals a mean fibre diameter of $\sim 360 \mathrm{~nm}$. TEM images of the fibres are shown in Fig. $1 \mathrm{~b}$ and indicate the presence of the nanotubes aligned along the fibres axis.
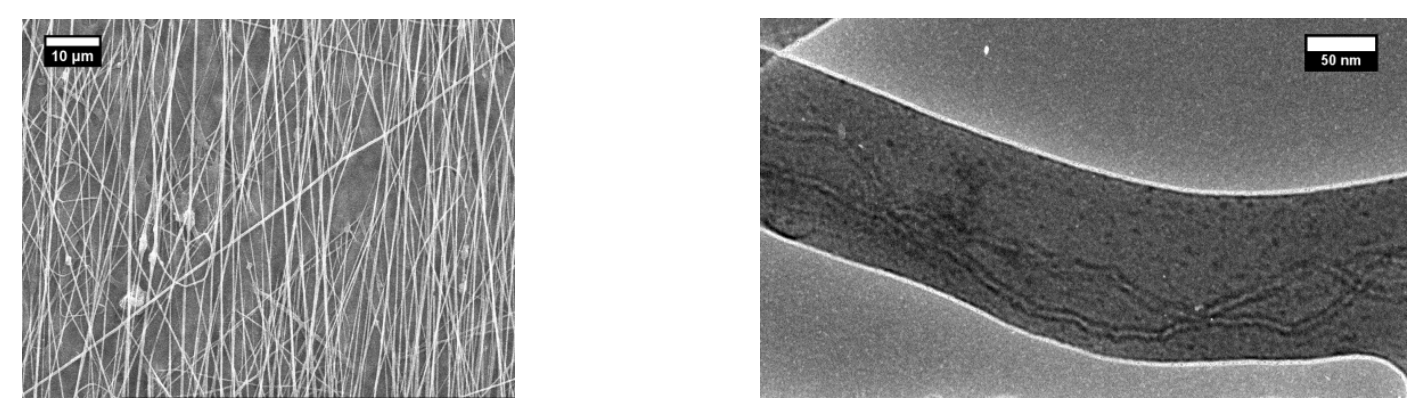

Fig 1. a) SEM micrograph of electrospun PEO fibres containing 5\% wt MWCNT b) TEM micrograph showing the MWCNT embedded in the fibres 


\section{Concept}

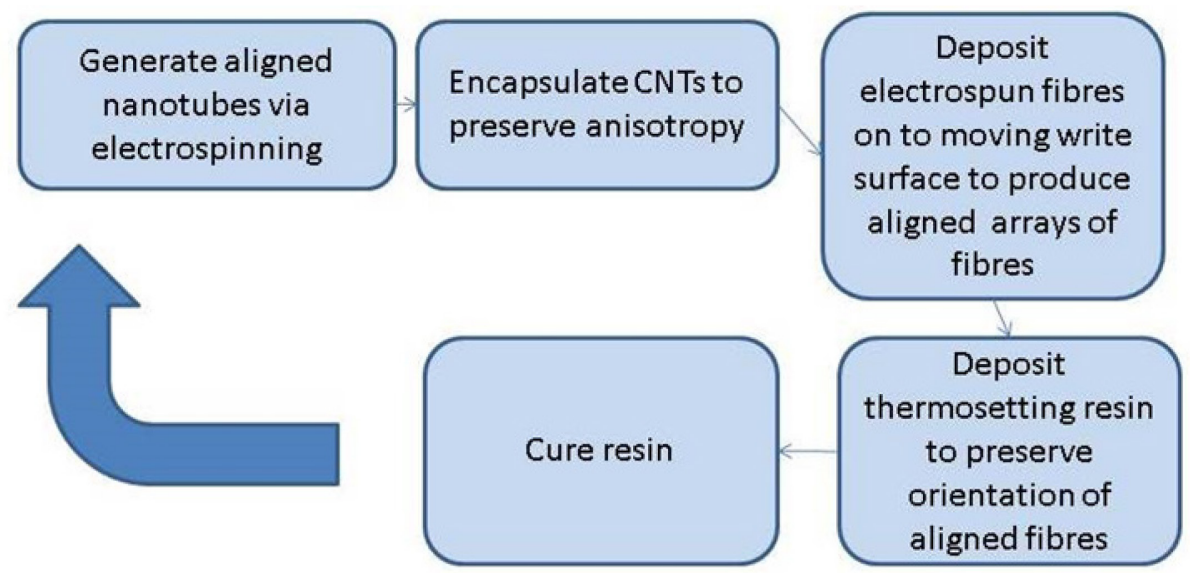

Fig 2. The key steps involved in the DDM production of nanocomposites based on a photocrosslinkable resin MWCNT

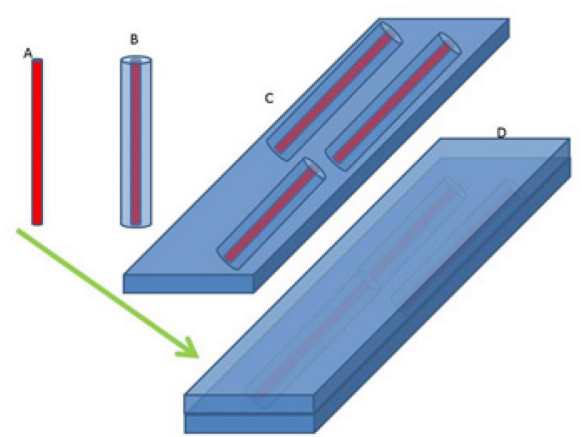

Fig 3. Schematic of the key steps shown in Figure 2 (a) Production of aligned MWCNTs using Electrospinning; (b) Encapulsation of aligned MWCNTs to preserved alignment and to facilitate manipulation; (c) Deposition of encapsulated aligned MWCNTs on to a surface using electrospinning; (d) Encapsulaation of the aligned arrays of electrospun fibres using the crosslinkable resin

The steps shown are largely practical in nature and underline the challenges in dealing with nanoscale objects - how to handle. The route chosen here both solves that challenges and how to preserve the alignment in the composite.

System Design. We have designed a system to incorporate the electrospinning system into an automated printing process for the fabrication of nanocomposite fibres and their incorporation into resins and described above. A schematic of all steps in the process is given in Fig. 2.

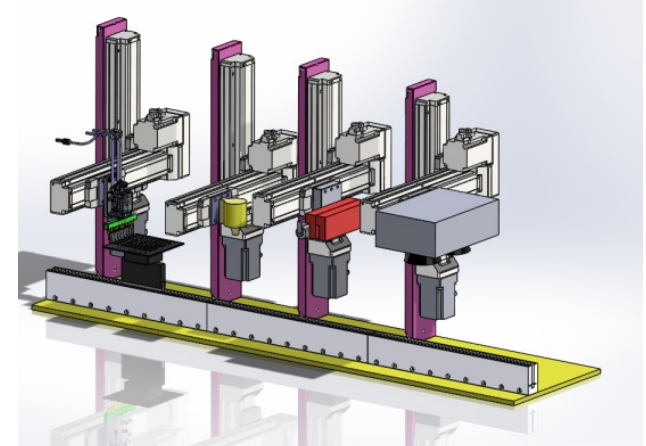

Fig. 4. Production process for resins containing nanocomposite fibres showing all stages in the process 
Fig. 3 shows step 1 of the process which consists of an array of electrospinning needles filled with $\mathrm{PEO} / \mathrm{MWCNTs}$ solutions. The fibres are spun and collected onto a surface positioned on a high speed table, capable of rapid back and forth movement along the y-axis (parallel to the track in Fig. 2 ) with speeds of several metres per second. The rapid back and forth motion aids in the alignment of the fibres as they are being deposited. The table is capable of rotating through the $x-y$ plane from $0-360^{\circ}$, thereby allowing deposition of fibres with orientations varying from 0 to $90^{\circ}$ angles with respect to the initial fibre layer. The deposition of fibres in multiple orientations will allow the exploitation of the improved mechanical properties in the more than one direction. In order for a layer-by-layer level of deposition, a z-axis control is incorporated onto the array of needles for height adjustments and control of the needle tip to collector distances.
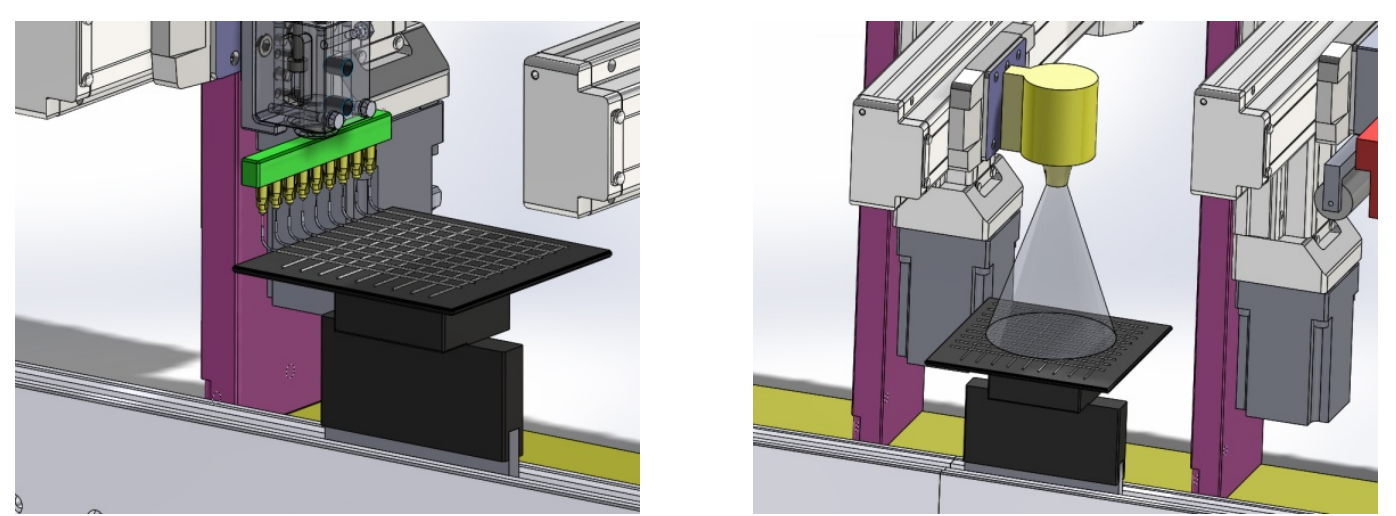

Fig. 3. Magnification of Fig. 2 focussing on step 1 of process. A multi-spinneret electrospinning array. b) Magnification of Fig. 2 focussing on step 2 of process. Spray head to apply photo-curable resin

Once the desired layer of nanocomposite fibres has been deposited, the automated process moves onto stage 2, where a print head deposits a layer of UV photo-curable resin on top of the polymer fibres (Fig. 3b). After the deposition of the resin the table progresses to stage 3 (Fig. 4) where a Teflon roller presses the curable resin down to a fine, uniform level and a combination of UV and IR light is applied in selected computer controlled areas using micro-mirrors [9, 10], curing the resin and fixing the orientation of the nanocomposite fibres within the photopolymer.
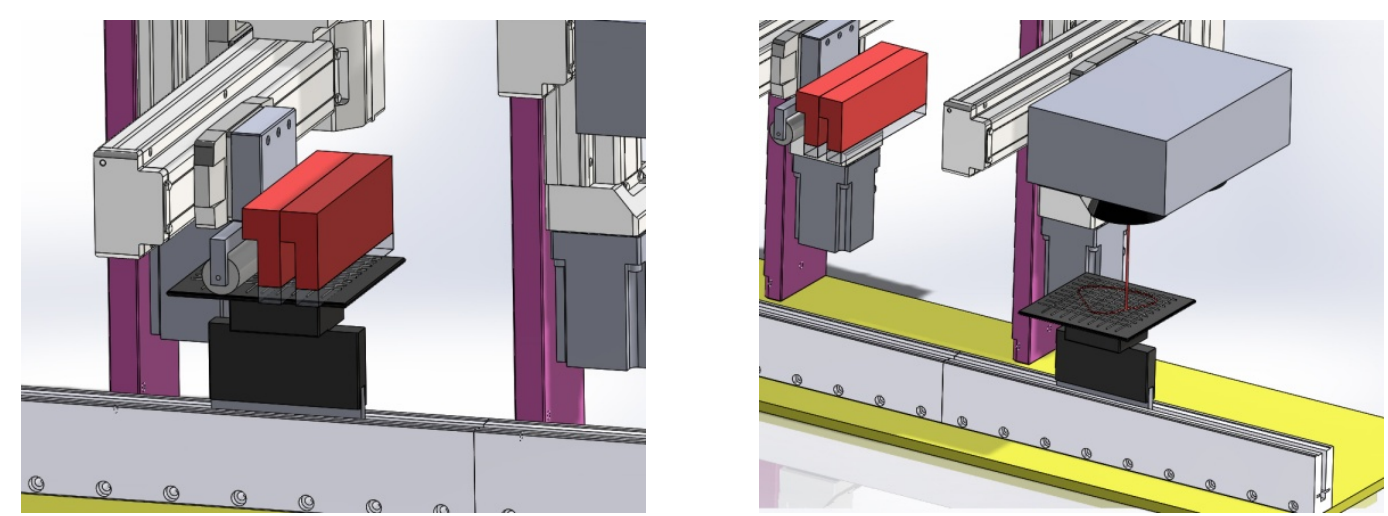

Fig. 4. Magnification of Fig. 2 focussing on step 3 of process. Roller to even resin, heaters and UV laser for curing of resins. b) Magnification of Fig. 2 focussing on the final step in the process. A laser capable of cutting the resin material into a desired shape

Steps $1-3$ are repeated as needed until the desired object dimension is achieved, after which in step 4, a CO2 laser is used to cut a desired shape of material (Fig. 4b).

Most literature that has investigated the incorporation of nanofibers into a resin often involves using layers or fibre bundles, laid by hand to which an epoxy is added and allowed to penetrate into the fibre mats. Alternatively fibres are mixed into the resin via mechanical stirring or sonication [10- 
12]. Here the process is automated and the direct control of the fibre deposition allows for orientations in a multitude of directions, enhancing the resins mechanical properties in these directions in a controlled fashion, rather than relying on a random mat orientation or being limited to a uniaxial enhancement through use of fibres oriented in one specific direction.

The system described above has focused on the use of PEO/MWCNT fibre composites, however this methodology will work with any electrospinnable polymer and filler particles. For example the nanofiller used can be replaced by sustainable and eco-friendly material such as nanofibrillated cellulose or crystalline nanocellulose; these particles have similar dimensions to those of carbon nanotubes [13]. There are already reports of where this material has been incorporated into the electrospinning process and has been shown to improve on the mechanical properties of the fibres produced. For example, Fortunato et al have incorporated nanofibrillated cellulose into PEO fibres prepared through electrospinning, observing a nearly 10 fold increase in the elastic modulus of the fibres compared to fibres prepared from pure PEO [14]. Other nanofiller particles that can be utilised are nanoclays such as halloysite which again have similar dimensions to that of the carbon nanotubes.

\section{Summary}

We have described an additive manufacturing process to prepare aligned nanocomposite materials embedded in an epoxy resin via exploitation of the anisotropy developed during the electrospinning process. A complete working system has been developed to automatically process the nanocomposite fibres and encase them in an epoxy resin to retain the specified orientation to enhance the mechanical properties of the resin.

\section{Acknowledgements}

MN acknowledges the support of a BOLASHAK scholarship from the Ministry of Education, Kazakhstan for her internship at the University of Reading. Part of this research leading to these results was funded by the European Union Seventh Framework Programme (FP7/2007- 2013) under grant agreement $n^{\circ} 263017$, Project "NanoCelluComp". Part of the work of AM, SDM and GRM was supported by P2020-PTDC/CTM-POL/7133/2014 FCT (Portugal). The electron microscopy was performed in the Centre for Advanced Microscopy at the University of Reading and we thank Matthew Spink for his help.

\section{Competing Interests}

A patent application has been granted by the Instituto Nacional da Propriedade Industrial of Portugal related to this work. There are no other conflicts of interest.

\section{References}

[1] K.V. Wong , A. Hernandez, A Review of Additive Manufacturing, ISRN Mechanical Engineering 2012 (2012) 1-10.

[2] G. Mittal, V. Dhand, K.Y. Rhee, S.-J. Park , W.R. Lee, A review on carbon nanotubes and graphene as fillers in reinforced polymer nanocomposites, Journal of Industrial and Engineering Chemistry 21 (2015) 11-25.

[3] M. Etcheverry , S.E. Barbosa, Glass Fiber Reinforced Polypropylene Mechanical Properties Enhancement by Adhesion Improvement, Materials (Basel) 5 (2012) 1084-1113.

[4] W.-I. Park, M. Kang, H.-S. Kim , H.-J. Jin, Electrospinning of Poly(ethylene oxide) with Bacterial Cellulose Whiskers, Macromolecular Symposia 249-250 (2007) 289-294.

[5] K. Iakoubovskii, Techniques of aligning carbon nanotubes, Open Physics 7 (2009)

[6] G.R. Mitchell, Electrospinning: principles, practice and possibilities, ed., 2015. 
[7] D.K. Shinde, A.D. Kelkar, Effect of TEOS electrospun nanofiber modified resin on interlaminar shear strength of glass fiber/epoxy composite, World Acad. Sci. Eng. Technol. Int. J. Mater. Metall. Eng 1 (2014)

[8] M. Nazhipkyzy, S.D. Mohan, F.J. Davis , G.R. Mitchell, Carbon nanotubes in electrospun polyethylene oxide nanofibres: A potential route to conducting nanofibres, Journal of Physics: Conference Series 646 (2015) 012007.

[9] P. Bartolo , G. Mitchell, Stereo-thermal-lithography: a new principle for rapid prototyping, Rapid Prototyping Journal 9 (2003) 150-156.

[10] C. Sun, N. Fang, D. Wu , X. Zhang, Projection micro-stereolithography using digital micromirror dynamic mask, Sensors and Actuators A: Physical 121 (2005) 113-120.

[11] H. Liao, Y. Wu, M. Wu, X. Zhan , H. Liu, Aligned electrospun cellulose fibers reinforced epoxy resin composite films with high visible light transmittance, Cellulose 19 (2012) 111-119.

[12] H. Fong, Electrospun nylon 6 nanofiber reinforced BIS-GMA/TEGDMA dental restorative composite resins, Polymer 45 (2004) 2427-2432.

[13] T. Guo, Z. Zhou, H. Guo, G. Xiao, X. Tang, M. Peng, Toughening of epoxy resin with functionalized core-sheath structured PAN/SBS electrospun fibers, Journal of Applied Polymer Science 131 (2014)

[14] C. Miao, W.Y. Hamad, Cellulose reinforced polymer composites and nanocomposites: a critical review, Cellulose 20 (2013) 2221-2262. 